\title{
Signal Processing Interpolation and Problem Conditioning Educational Workbench
}

\author{
Mónica Isabel T. da $\operatorname{Costa}^{1}$, Salviano Soares ${ }^{2} \&$ João Barroso $^{3}$ \\ ${ }^{1}$ EST - Informatics Department, Polytechnic Institute of Castelo Branco, Castelo Branco, Portugal \\ ${ }^{2}$ UTAD/ECT Engineering Department, Vila Real 5001-801, Portugal; IEETA, UA Campus, Aveiro 3810-193, \\ Portugal \\ 3 INESC TEC (formerly INESC Porto) \UTAD - University of Trás-os-Montes e Alto Douro, Vila Real \\ 5001-801, Portugal
}

Correspondence: João Barroso, INESC TEC (formerly INESC Porto) \UTAD - University of Trás-os-Montes e Alto Douro, Vila Real 5001-801, Portugal. E-mail: jbarroso@utad.pt

Received: September 24, 2013

Accepted: October 17, $2013 \quad$ Online Published: November 18, 2013

doi:10.5539/mas.v7n12p56

URL: http://dx.doi.org/10.5539/mas.v7n12p56

\begin{abstract}
This work presents a newer and more complete version of an educational tool to be used in signal processing interpolation-related subjects. Besides the consolidation of acquired theoretical knowledge, the tool allows now its users to apply three error geometry patterns, test minimum or maximum dimension signal reconstruction algorithms and problem conditioning through the analysis of the matrix spectral radius or the condition number: these new features gives the possibility to alter the problem definitions to the desired goal before the reconstruction begins. The time unit that measures the algorithms performance is $\partial(n \log n)$ thus independent from the machine's architecture. A video of the developed tool can be seen in: https://www.dropbox.com/s/t6yiiuy31ramxse/FILME_1.avi.
\end{abstract}

Keywords: signal reconstruction, educational tool, interpolation, oversampling, fast Fourier transform (FFT), condition number, matrix spectral radius, problem conditioning

\section{Introduction}

Nowadays, namely in engineering areas, there are a great amount of subjects that require a large and deeper knowledge of theoretical aspects related to a specific scientific area. Sometimes students don't really understand the need to have skills on important concepts and lack the perception to understand its utility or even if they really work. During the last decades, with the quick spread of information technologies, some new tools arose whose purpose is to ensure and consolidate the theoretical concepts taught in regular teaching classes. The main idea is to give students a way to experiment concepts taught in classes, as well as giving them the opportunity to test them in different scenarios. With these tools students and other kind of users can practice and enhance skills by themselves as self-taught persons.

In this paper a new version of the "Signal Processing Interpolation Educational Tool" (SPIEW) is presented (Costa et al., 2012). In the first version we propose a new and easy-to-use tool which will help students consolidate concepts of signal processing and complement their knowledge by enabling graphical results after using complex signal reconstruction algorithms. The tool permits to achieve calculation in just one hit or step by step through the iterative reconstruction refinement process and the assessment of the reconstructed signals.

Now we present an improved version of SPIEW. Its main new features are: the possibility to apply three error geometry patterns (interleaved, random and burst); control the redundancy of the signals by applying a proper oversampling factor, $r$, in the band limiting operation; test two different classes of signal reconstruction algorithms and the usage of the iterative and direct calculation methods; the previously set up the problem conditioning through the analysis of the spectral radius, $\rho(S)$, of the iteration matrix (in the case of iterative methods are used) and the computation of the condition number, $k(A)$, in the case of the direct methods are used. In this way, its user can employ these parameters to know in advance if the problem will converge to an accurate solution, even before running one of the reconstruction methods available. A previous analysis of key parameters permit to chose the most suitable method to be used in the signal reconstruction test scenarios. 
Since all the chosen methods use the FFT computation, $\partial(n \log n)$, the time necessary to compute a FFT is used as reference. So, the performance is measured through a relative Time Unit (TU) that is the time to compute a FFT in a particular machine.

\section{Reconstruction Algorithms}

The signal reconstruction algorithm used in SPIEW was the discrete version of the Papoulis algorithm (Papoulis, 1975): the Papoulis Gerchberg algorithm (Ferreira, 1994a; Jones, 1986). A thorough description of this maximum dimension algorithm can be found in (Costa et al., 2012). The Papoulis algorithm is still available to run but a new minimum dimension reconstruction algorithm was included like the one used in (Neves et al., 2008). Two different methods can be used to implement this algorithm: iterative and non-iterative calculation. We also call the non-iterative method the direct calculation method. This way students can test and compare maximum and minimum dimension algorithms with direct and iterative formulations. In the next subsections the minimum dimension algorithm is presented.

\subsection{Minimum Dimention Algorithm}

This subsection describes a minimum dimension algorithm which also requires band-limited signals of finite-dimension similarly to the Papoulis Gerchberg algorithm.

Thus the signal

$$
x=B x
$$

where $B$ is a band-limiter operator matrix (Ferreira, 2-1994).

The minimum dimension algorithm is a system of only $l$ equations corresponding to the $l$ unknown samples when the total number of samples is $n$ and $n>l$.

To establish the basic concepts, the specific case of an original signal $x[n]$ with length $n=5$ is used, i.e., $x[n]=\left\{x_{1}\right.$, $\left.x_{2}, x_{3}, x_{4}, x_{5}\right\}$.

For this signal, Equation (1) becomes

$$
\begin{aligned}
& x_{1}=B_{11} x_{1}+B_{12} x_{2}+B_{13} x_{3}+B_{14} x_{4}+B_{15} x_{5} \\
& \cdots \\
& x_{5}=B_{51} x_{1}+B_{52} x_{2}+B_{53} x_{3}+B_{54} x_{4}+B_{55} x_{5}
\end{aligned}
$$

For reconstruction purposes let us assume that the $2^{\text {nd }}$ and $4^{\text {th }}$ samples of $x[n]$ are lost. Then, because only the unknown samples are to recover, the set of equations, Equation (2), will be limited to those including the unknown samples. In each of these equations, we are interested in separating the right side terms of Equation (2) containing the unknown samples $\left(x_{2}, x_{4}\right)$ from those containing the known ones. These yields,

$$
\begin{aligned}
& x_{2}=B_{21} x_{1}+B_{22} x_{2}+B_{23} x_{3}+B_{24} x_{4}+B_{25} x_{5} \\
& x_{4}=B_{41} x_{1}+B_{42} x_{2}+B_{43} x_{3}+B_{44} x_{4}+B_{45} x_{5}
\end{aligned}
$$

which is equivalent to

$$
\left[\begin{array}{ll}
x_{2} & x_{4}
\end{array}\right]=\left[\begin{array}{ll}
B_{22} & B_{24} \\
B_{42} & B_{44}
\end{array}\right]\left[\begin{array}{l}
x_{2} \\
x_{4}
\end{array}\right]+\left[\begin{array}{lll}
B_{21} & B_{23} & B_{25} \\
B_{41} & B_{43} & B_{45}
\end{array}\right]\left[\begin{array}{l}
x_{1} \\
x_{3} \\
x_{5}
\end{array}\right]
$$

Let us denote by $u$ the subset of the original signal $x[n]$ which contains the unknown values. In this case, $u=\left\{x_{2}, x_{4}\right\}$ is of cardinality $l=2$. Also, let us define $U=\left\{i_{1}, \ldots, i_{l}\right\}$ as the set of subscripts of $l$ unknown samples in $x[n]$. In the present case, $U=\{2,4\}$. Therefore, Equation (4) can be written as

$$
x_{i}=\sum_{j \in U} B_{i j} x_{j}+\sum_{j \notin U} B_{i j} x_{j} ; \quad i \in U
$$

or, in matrix form

$$
u=S u+h,
$$

where $S$ is a $l \times l$ principal submatrix of $B$, as defined in Equation (4), and $h$ is the (n- $l$ ) dimensional vector in the second sum of Equation (5), which is a linear combination of the known samples of $x[n] . S$ is the system matrix 
(Ferreira, 1994b). Theoretically, Equation (6) has a unique solution regardless the number and distribution of the lost samples.

On the one hand, by successive operations, Equation (6), becomes equivalent to

$$
\begin{gathered}
u=S u+h \\
u=(I-S)^{-1} h,
\end{gathered}
$$

and a basis to implement the non-iterative method is so established. On the other hand Equation (6) suggests the needed formula to the iteration process

$$
u^{(i)}=S u^{(i-1)}+h,
$$

for the case where a non-relaxation method is used. Then $u^{(i)}$ is obtained at iteration $i$ and the solution is given by the limit

regardless of $u^{(0)}$.

$$
u=\lim _{k \rightarrow \infty} u^{(k)}
$$

Direct calculation of $u$ as given in Equation (7) has the advantage of being done in one single step and gives an exact solution, providing that $(I-S)^{-1}$ exists. The drawback is that in case $(I-S)^{-1}$ does not exist or the calculation is not possible and no reliable approximation is possible. In practice, there are several factors which may lead to serious difficulties in calculating the inverse of $I-S$. For example, if one of the eigenvalues of $S, \lambda_{i}(S)$, is close enough to the unity, then the computation of $(I-S)^{-1}$ may become very difficult, even impossible, which leads to an ill conditioned problem. In such cases an iterative method is advantageous because it may be used to circumvent this difficulty and to find a relatively accurate approximation for the solution, $u$. Despite the fact of having an ill conditioned problem, in direct calculation method such problem is impossible to solve whereas in iterative methods an approximation is always possible to be found, though its accuracy may not be very high. The conditions under which these equations provide a solution for $u$ can be found in (Ferreira, 1994a) and will be discussed in the next subsection.

\section{Convergence Analysis}

It is necessary that $(I-S)^{-1}$ exists or the iterative processes converge to find a problem solution. It is demonstrated in (Ferreira, 1994a) that for low pass signals of dimension $n$ with $s$ known samples and $q$ nonzero harmonics, reconstruction iterative algorithm converges if:

$$
(n-q) \geq(n-s)
$$

which is equal to

$$
q \leq s .
$$

It is important to refer that $q=2 m+1$ where $m$ is the sample bandwidth of the Low Pass Filter (LPF) (Costa et al., 2012). So Equation (11) can be rewritten as follows:

$$
s \geq 2 m+1 .
$$

which establishes a sufficient condition on the density of the known samples. More precisely, the number of known samples $s$ must exceed twice the number of nonzero harmonics. For low pass signals, the condition is consistent with the well-known classical sampling theorem.

The sufficient condition expressed by (12) is obviously necessary too. After all, low-pass signals with $q=2 m+1$ nonzero harmonics are described by $2 m+1$ parameters, and therefore one cannot expect to reconstruct them from less than $2 m+1$ samples.

In order to use the algorithms available in the new tool it is necessary that all signals are band limited. This means that Equation (12) establishes a necessary condition in our tool. Because only low pass signals are used, the necessary condition is met.

For the specific case of the Papoulis-Gerchberg algorithm, a relaxation constant, $\mu$, is introduced to improve the convergence rate, as stated in the previous tool version. In (Ferreira, 1994a) it is demonstrated that the algorithm converges if both conditions are satisfied: $0<\mu<2$ and $s \geq 2 m+1$ Equation (12). Because we have considered $\mu=1$ and used low pass signals the iterative process converges.

Regarding minimum dimension method, the characterization of the system matrix, $S$, is fundamental to infer 
about the existence of solution to the problem formulated in Equation (2). Namely its eigenvalues, $\lambda_{i}(S)$, and in particular its spectral radius, $\rho(S)$, condition the problem. $\rho(S)$ values close to 1 (the unity) make the computation of $(I-S)^{-1}$ very difficult, even impossible. Theoretically, the condition $\rho(S)<1$ would always guarantee the solution possible. However, due to the some computational representation constraints, small variations on input values can cause big variations on the output and cause the problem to be ill conditioned. In these cases a new implemented parameter must to be taken into account: the condition number of $(I-S)^{-1}$, presented latter below.

According to these conditions, it is possible to put a reconstruction problem into a point such that it is well conditioned. It is known that the eigenvalues of the system's matrix $S$ depend on the distribution of the missing samples. In particular, its spectral radius is more likely to be unitary for burst distributions rather than for equidistant missing samples (Ferreira, 1994c). In the case of signal reconstruction it is interesting to note that, if the distribution of the missing samples $U=\left\{i_{0}, i_{1}, \ldots, i_{l-1}\right\}$ is equidistant by some fixed integer $p \geq 1$, that is, $U=$ $\left\{i_{0} p, i_{1} p, \ldots, i_{l-1} p\right\}$, then the eigenvalues $\lambda_{i}$ of $S$ are given by $\lfloor r p\rfloor / p$, i.e.,

$$
\frac{\lfloor r p\rfloor}{p} \leq \lambda_{i}(S) \leq \frac{\lceil r p\rceil}{p} \leq 1
$$

where $\lfloor r p\rfloor$ denotes the greatest integer less than or equal to $r p$ and $\lceil r p\rceil$ denotes the smallest integer equal or greater than $r p$. In the particular case of $r=\lfloor r p\rfloor / p$, the eigenvalues of $S$ are all the same $\lambda_{i}(S)=r, \forall i$.

Given the above analysis, it is possible to put the problem into a well-conditioning point by properly selecting the gap between the missing samples. Then it is possible to put $\lambda_{i}(S)$ close to either $r$ or its multiples, regardless of the number of missing samples. By using an appropriate choice of the oversampling and interleaving factors, $r$ and $p$, respectively, it is possible to put $\lambda_{i}(S)$ less enough than the unity in order to control the reconstruction accuracy and processing speed (i.e., such that $m \times r$ is an integer).

Figure 1 shows the maximum and the minimum eigenvalues of $S$ as a function of the interleaving factor, $p$, for a given bandwidth, defined by $r=0.6$. As the figure shows, greater values of $p$ lead to better-conditioning problems because $\lambda_{\max }$ decreases as $p$ increases. Also, when the product $m \times r$ is an integer, all eigenvalues are equal since they are $\lambda_{i}(S)=r$, as stated before. In Figure 1 , this occurs for $p=5$ and $p=10$.

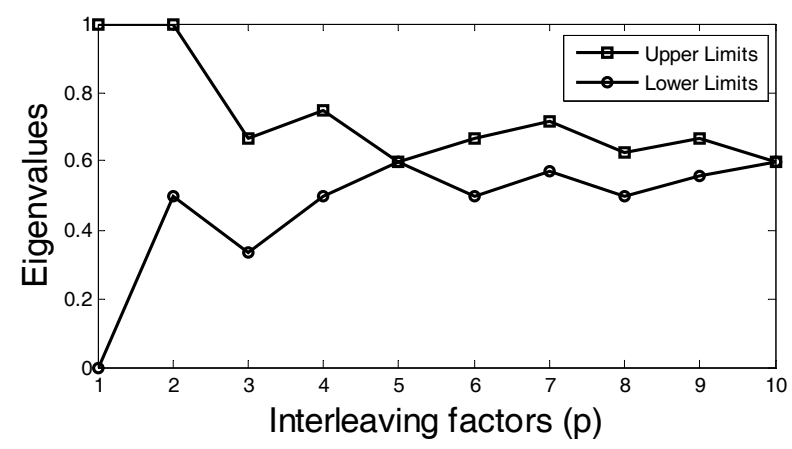

Figure 1. Spectral radius vs. interleaving factor, $r=0.6$

To better understand and control the problem conditioning, in the presented tool, some new parameters are calculated: the spectral radius of the $S$ matrix, delimited in Equation (13) and the Condition Number of the (I-S) matrix (Equation (14)) (Golub \& Van Loan, 1996; Kincaid \& Cheney, 2002). Through the analysis of both values, and also the convergence condition, Equation (12), the user can know in advance if the reconstruction algorithm will converge to an accurate solution or not. Therefore through the properly chosen condition point the signal reconstruction is guaranteed.

In practice, when $\rho(S)$ tends to be very near or even equal to the unity, the problem may become ill-conditioned, even if it is theoretical well-conditioned due to the fact that $\rho(S)<1$. Condition numbers near or above $10^{15}$ do not generally lead to well-posed problems, even with double precision arithmetic (64-bit floating point, in many machines). The condition number of matrix $A, k(A)$, is calculated according the equation presented in (Kincaid \& Cheney, 2002): 


$$
k(A)=\|A\|\|\| A \|^{-1} \geq \frac{\left|\lambda_{\text {max }}(A)\right|}{\left|\lambda_{\text {min }}(A)\right|} .
$$

\section{Developed Tool}

This tool was developed using Matlab (Maltlab, 2012) and is to be used with band limited signals. Signals to be treated in this version are: frame sequences or still images. In the first version of SPIEW there was only one signal corruption method, the interleaved decimation and only a maximum dimension algorithm, the Papoulis Gerchberg. An improvement was the addition of two geometry patterns error and a new reconstruction algorithm. This new version allows the signal corruption both in a random and contiguous or burst way: the Papoulis Gerchberg is also available. The next subsections describe each of the stages that constitute a complete cycle of using the tool.

\subsection{Extract Signals Operation}

To start using the SPIEW application the user must push the "Open File" button to choose a type of signal: as previously referres it can be the first frame of a sequence or a still image, here we have used Multiview Video plus Depth (MVD) 3D images (Rusanovskyy et al., 2013). This is the only active button when the user starts the tool. The rest of the buttons will be activated sequentially to force the user to perform all the correct actions in the correct order. In Figure 2 we can observe the first interface of the application after doing the opening file operation.

After the chosen signal is open and displayed, some information regarding it is showned. If the user has chosen the sequence frames to work with the "Play" button is enabled. In the case of a still image this button will not be activated. To perform the signals extraction form each type of signal the user must clik the "Extract Signals" button. Each signal is made by: all the values corresponding to the same pixel along the frame sequence or each line of the still image. When this button is pressed the signals extraction is done and a progress bar shows the user the remaining time to complete the task (Figure 3).

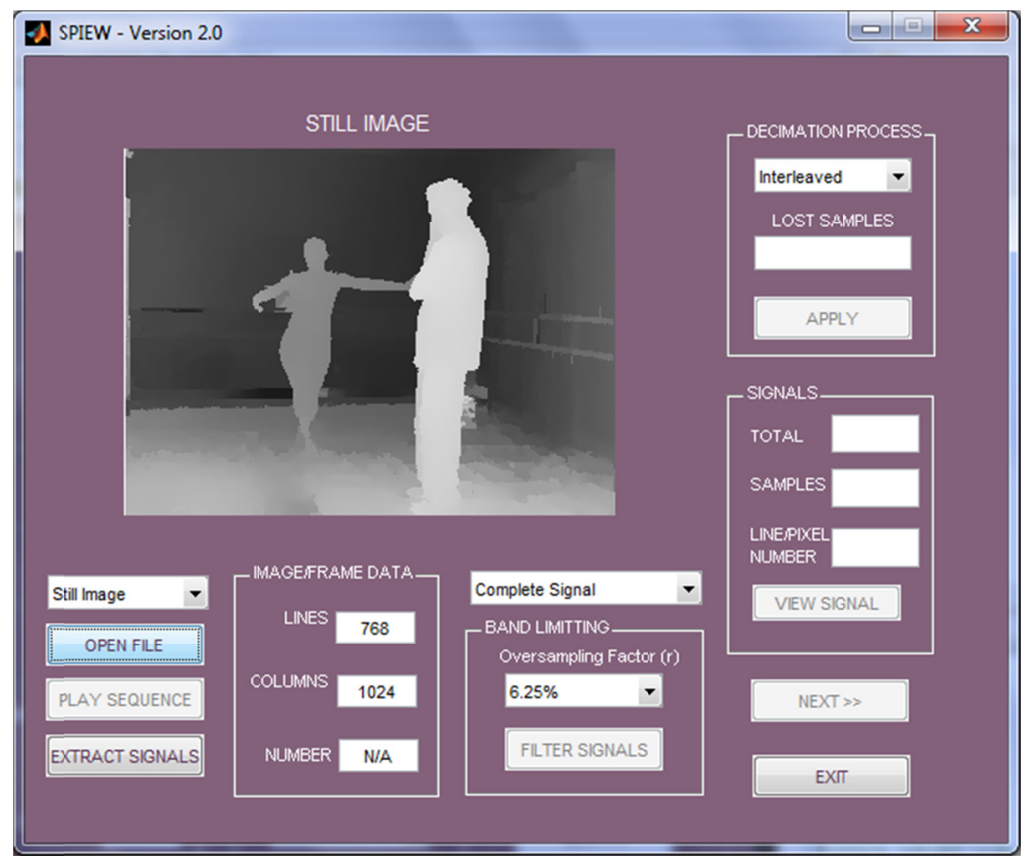

Figure 2. SPIEW initial interface, still image file

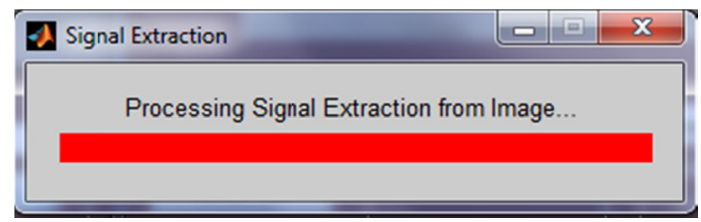

Figure 3. Signal extraction progress bar 
It is now available to the user the number of signals extracted from the entire sequence, as well as the total number of samples of each extracted signal.

\subsection{Band Limitting Operation}

After the signals extraction the user can now perform the band limiting operation for each of the extracted signals. This is a necessary condition to all the reconstruction algorithms available in this tool. For that the user must chose the value of the oversampling factor, $r$. It indicates the percentage of redundancy that the signals will have after the filtering operation and also limits the number of samples that can be lost afterwards. To initiate this process the user must press the "Filter Signals" button. Subsequently a progress bar appears showing the estimated time to finish this operation. Eventually a message box shows the Low Pass Filter Bandwidth that corresponds to the $r$ chosen value (Figure 4). This is the value of $m$ to verify Equation (12).

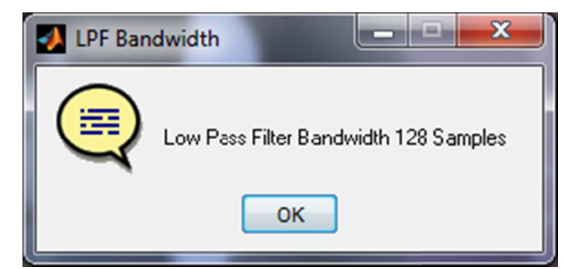

Figure 4. Band limiting operation - low pass filter bandwidth

\subsection{Corruption Operation}

The user is now ready to perform the corruption of the signals. As mentioned before, in the previous version of the tool, only one error pattern geometry was available to apply: the interleaved decimation. This new version has three possible error geometry patterns: interleaved, random and contiguous decimation (Figure 5). This will allow the user to compare the reconstruction algorithms performance in different corruption scenarios.

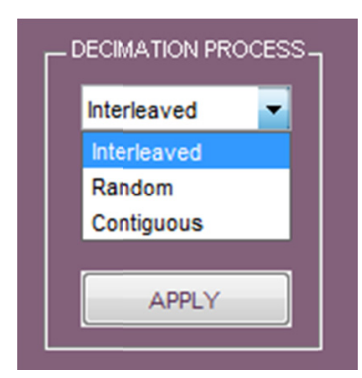

Figure 5. Decimation process, available methods

In the case of the interleaved decimation corruption the user must chose a value for the jump, as shown in Figure 6 . He must chose values among 2, 4 or 8 . After choosing the desired value to start the decimation process, the user must press de "Apply" button.

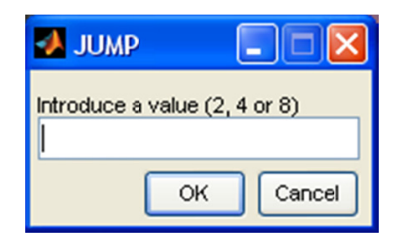

Figure 6. Choosing the jump value for the interleaved decimation method

In this particular case (interleaved decimation) the number of lost samples is automatically calculated and filled in when the decimation process is terminated. In the other two cases (random and contiguous) the user must choose how many samples he wants to lose in the decimation process (Figure 7). If the user wants to visualize the results of both the band limiting and the decimation operations, he must choose a specific pixel by its number and press the "View" button. A figure with the original, band limited signal (observed signal) and a decimated 
version of that signal appears (Figure 8).

It is important to refer that both band limiting and decimation operations are performed for all signals extracted from the video sequence or still image.

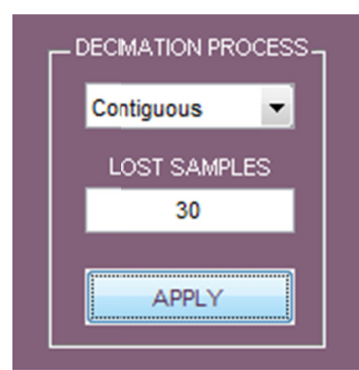

Figure 7. Contiguous decimation, 30 lost samples

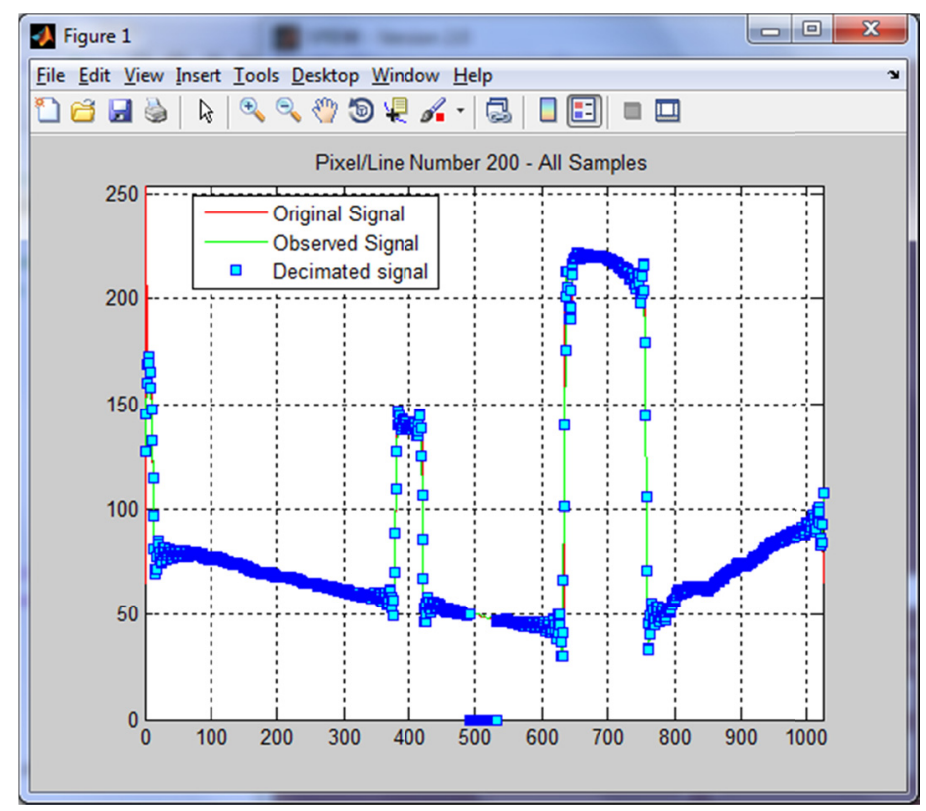

Figure 8. Original, Observed and Decimated versions of the signal corresponding to line number 200

\subsection{Convergence Analysis and Reconstruction}

We are now ready to move into the next interface of SPIEW. To do so the user must press the "Next" button in the current interface. The reconstruction window appears. Here the user must choose what reconstruction algorithm is to run through a pop-up menu with three options: Papoulis-Gerchberg, Minimum Dimension, Direct method or Minimum Dimension, Iterative method (Figure 9).

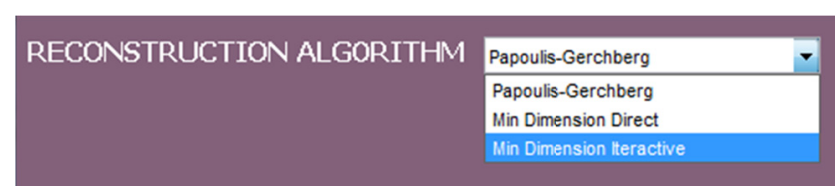

Figure 9. SPIEW, choose the reconstruction algorithm

Before starting the reconstruction of the chosen signal, the user is now able to see the problem conditioning data (spectral radius and condition number). It is through the analysis of these two values that the user will know in advance if the chosen algorithm and method will converge. To visualize these parameters, the user must press the Problem Conditioning button (Figure10). Here the spectral radius and condition number concerning the problem under study are exhibited. 


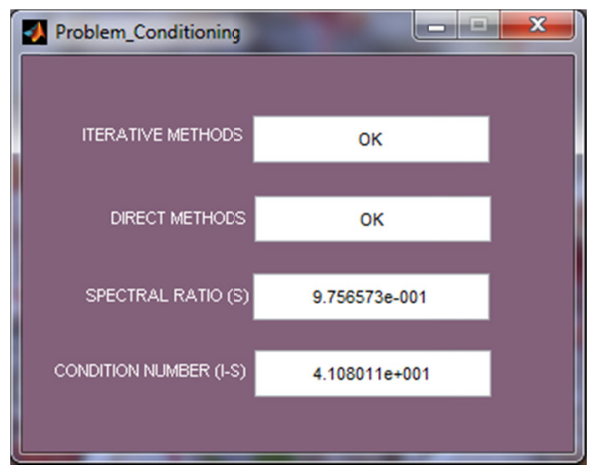

Figure 10. Problem conditioning

After analysing these values the user knows if the problem will converge to an accurate solution or not. He will also know which algorithm to choose to obtain an accurate solution. As mentioned in the previous section for iterative methods, a spectral radius smaller enough than the unity guarantees in practice the iterative algorithms' convergence. For direct methods a condition number smaller than $10^{15}$ guarantees a solution to the problem. More details about this issue will be presented in the Test and Results section.

After choosing the reconstruction algorithm the user must choose which signal we want to reconstruct and in the case of the iterative methods, he must also indicate the maximum number of iterations. To start reconstruction the user must press the "Run" button. After the signal reconstruction, besides the reconstruction results, including the reconstructed signal view, the user can also see the signal's energy and power. This will allow the comparison of the reconstruction of signals, or even signal segments, with more or less energy.

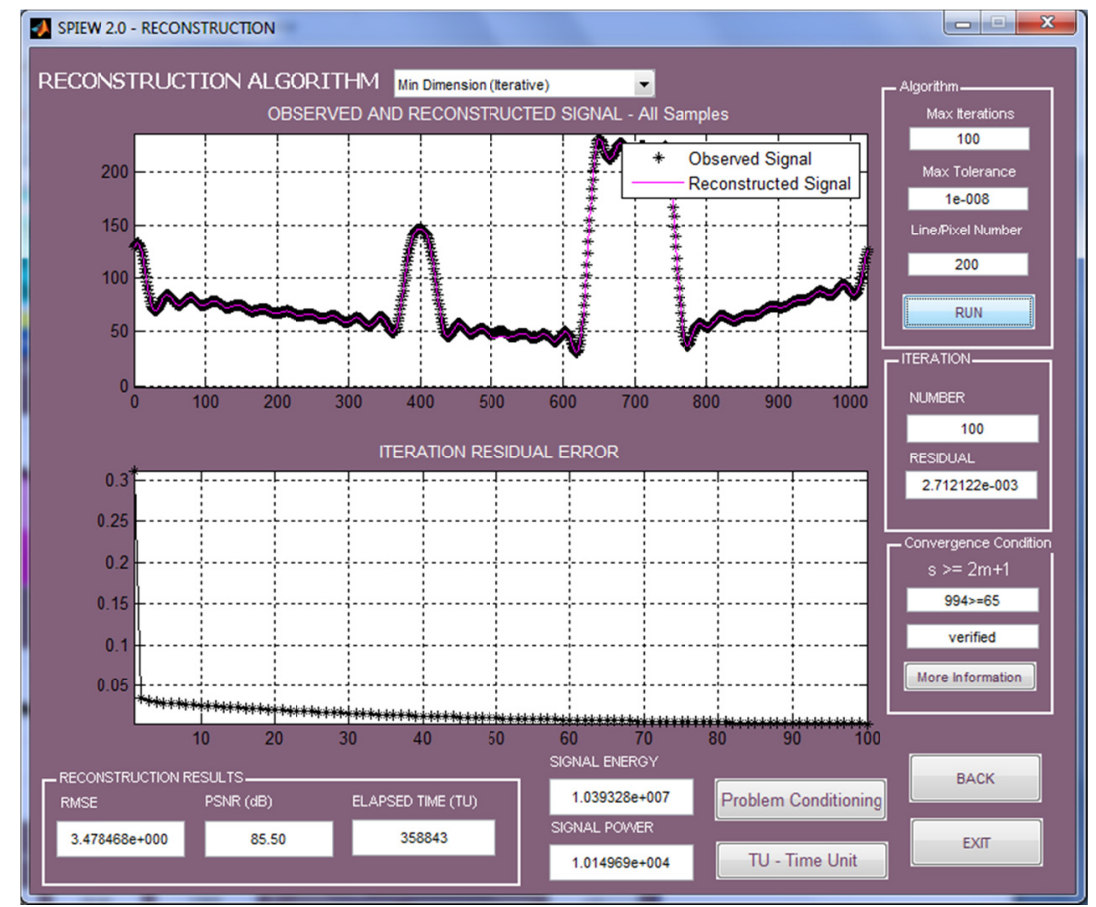

Figure 11. Reconstruction results using the Minimum Dimension, Iterative method (Line 200)

After the signal reconstruction has started, the window shown in Figure 11 allows the user to visualize the iteration number, its residual error and if the convergence condition is verified. There is also an explanation button to help the user to understand the expression used to verify the convergence. It is the "More Information" button in the convergence condition area (Figure 12). 


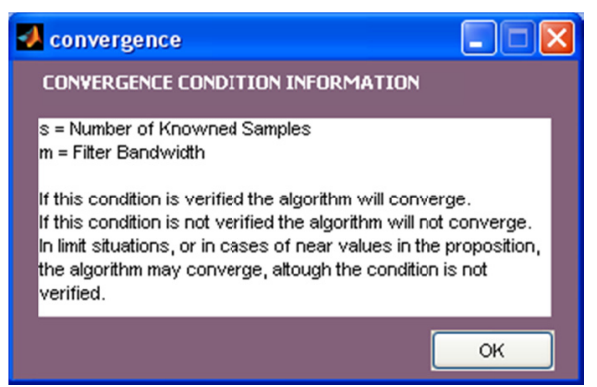

Figure 12. Convergence condition information

At the end of the reconstruction several parameters to help the user to evaluate the reconstruction and compare the available algorithms are calculated. Those parameters are: the Root Mean Squared Error (RMSE), the Peak Signal to Noise Ratio (PSNR) and the Elapsed Time measured in TU. By pressing the "TU - Time Unit" button the user can access this information (Figure 13). The tool also shows a graphic of the Residual Error calculated in each iteration (herein called the Iteration Residual Error). In it the user can see if the chosen algorithm tends faster to the maximum tolerance.

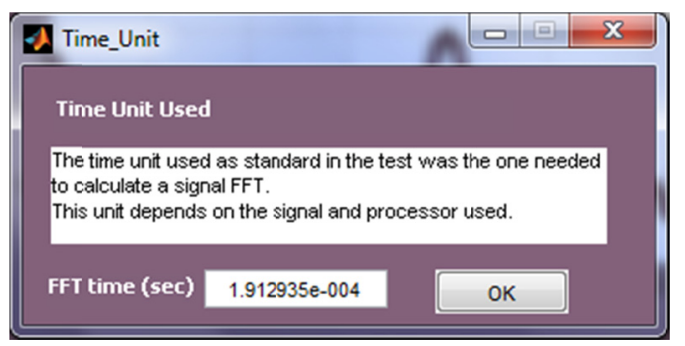

Figure 13. TU, time unit used to compare the reconstruction algorithms results

When the user chooses the Minimum Dimension algorithm, Direct method, there are parameters that are not needed or calculated, such as the maximum number of iterations, the iteration number and its residual error, and instead of the Iteration Residual Error graphic, only observed and reconstructed signals are showed (Figure 14).

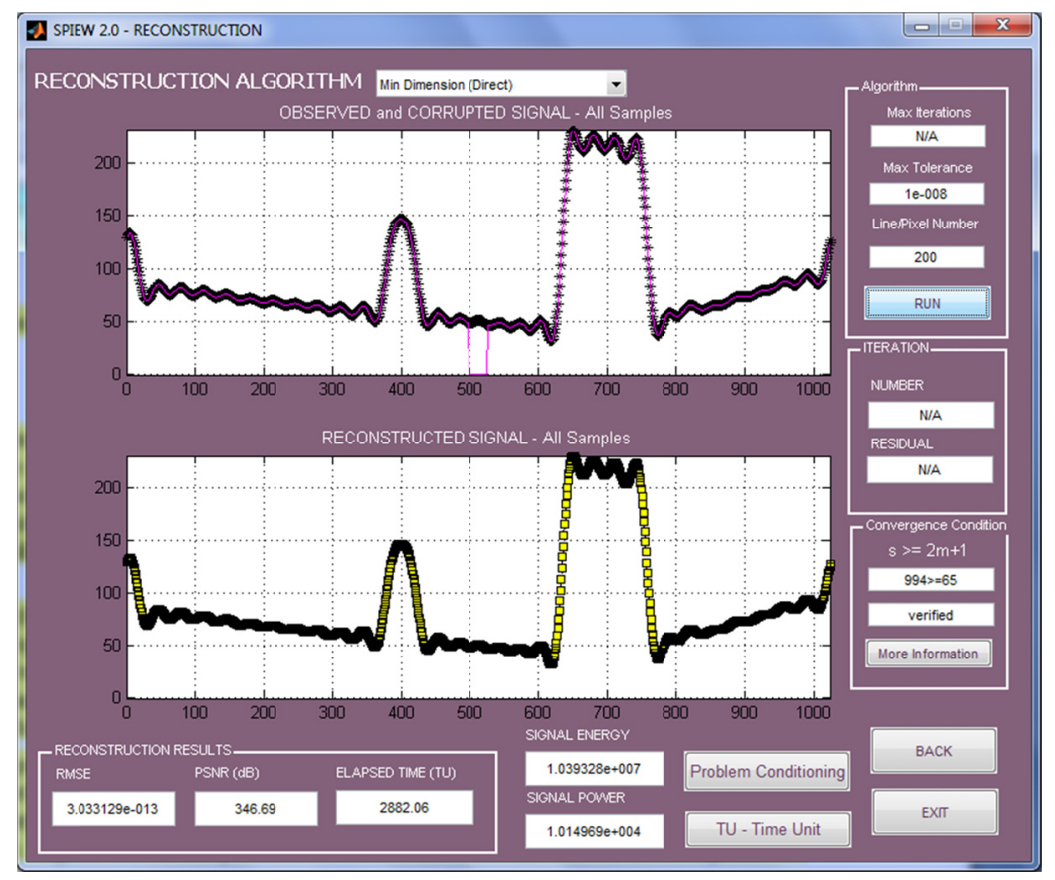

Figure 14. Reconstruction results using the Minimum Dimension algorithm, direct method, to reconstruct line 


\section{Tests and Results}

SPIEW was tested several times in order to better explain the diverse contents that it addresses to its users. Still during its development some opinions of colleagues and students were taken into consideration to make this tool more effective and user-friendly. For example, its GUI was altered quite a few times in order to comply with the obtained feedback. Concerning the SPIEW features, first the three error geometry patterns were applied and tested. Clearly the most unfavourable case to the reconstruction efficiency is the contiguous (burst) lost of samples.

Through those tests we concluded that the convergence condition must be verified to guarantee the algorithms' convergence. Furthermore a spectral radius less than 1, preferably around 0.5 , will ensure the well functioning of the iterative methods (convergence rate, accuracy, etc.). But in the specific case of the direct method the condition number is a key parameter to infer about how acceptable a solution using this method is. Values lower than $10^{15}$ lead to accurate solutions even when the iterative methods no longer converge.

In the following figures we present the results obtained from the tests achieved with $r=12.5 \%$ and a burst error geometry pattern for the lost of samples. It is important to refer that the burst, in this time, is located in the middle of the signal. The purpose is to compare different cases resulting from different number of lost samples by increasing the burst size. Figure 15 shows the problem condition values for the case of 10 contiguous lost samples.

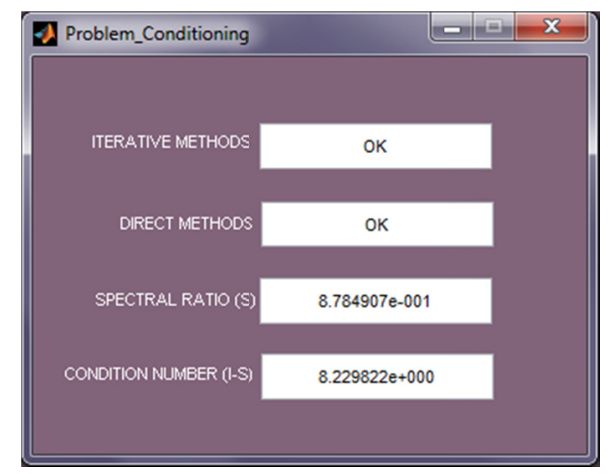

Figure 15. Problem conditioning values for 10 contiguous lost samples

Through the analysis of both spectral radius and condition number we conclude that both iterative and direct methods will converge. In Figure 16a and Figure 16b, the results obtained using both methods are shown.

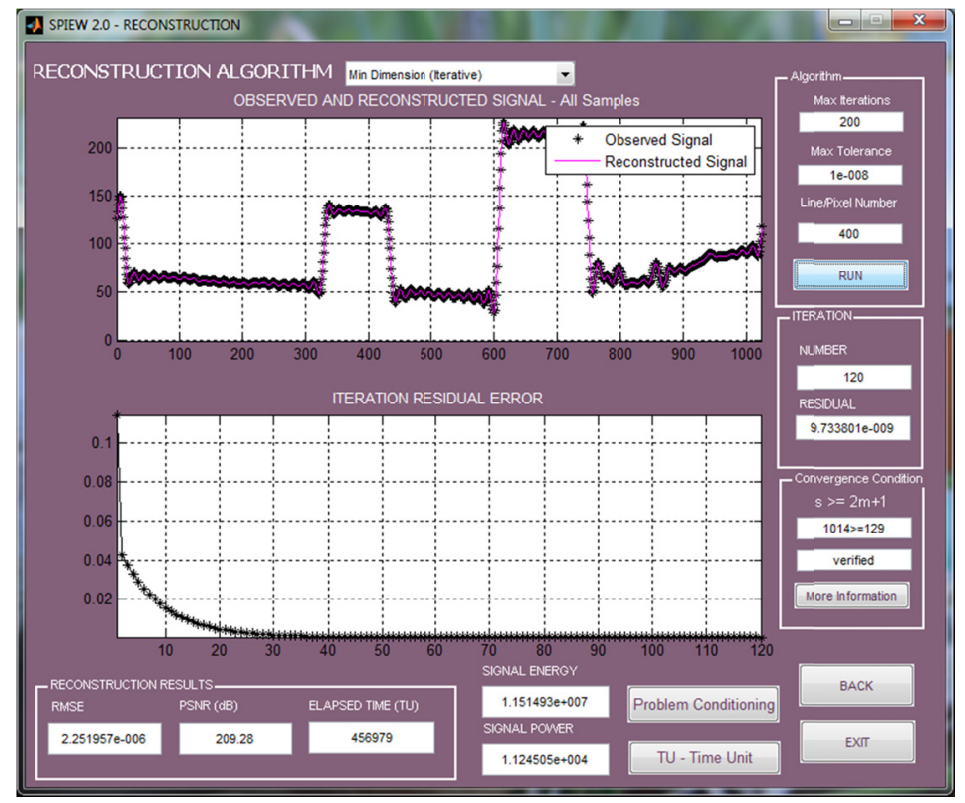

Figure 16a. Reconstruction results using the Minimum Dimension algorithm, Iterative method (Line 450) 


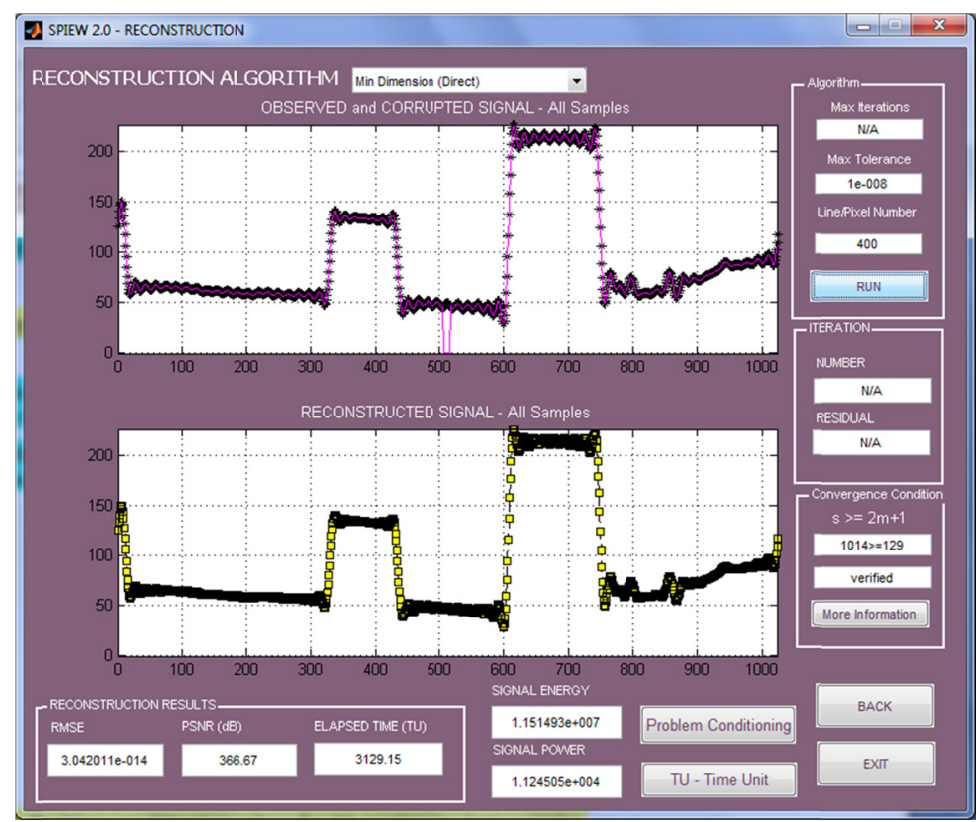

Figure 16b. Reconstruction results using the Minimum Dimension, Direct method (Line 450)

The next case results from increasing the number of the lost samples to 60 . Figure 17 shows the problem condition values that permit to achieve the respective convergence analysis.

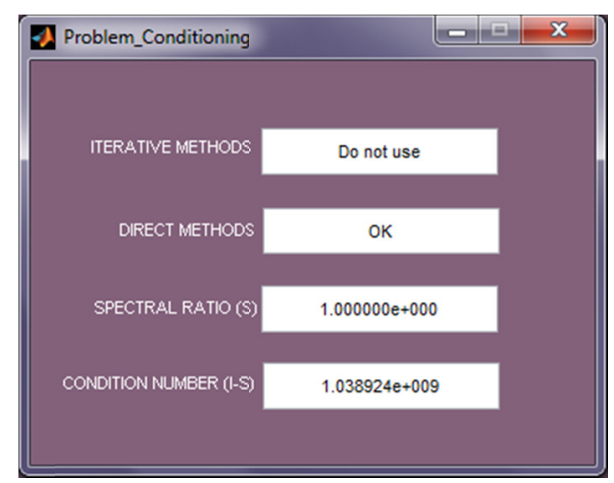

Figure 17. Problem conditioning values for 60 contiguous lost samples.

Clearly the iterative methods will no longer converge to an accurate solution, since spectral radius, $\rho=1$. However, it is possible to see that by using the direct method we will be able to obtain a solution, as shown in Figure 18a and Figure 18b. 


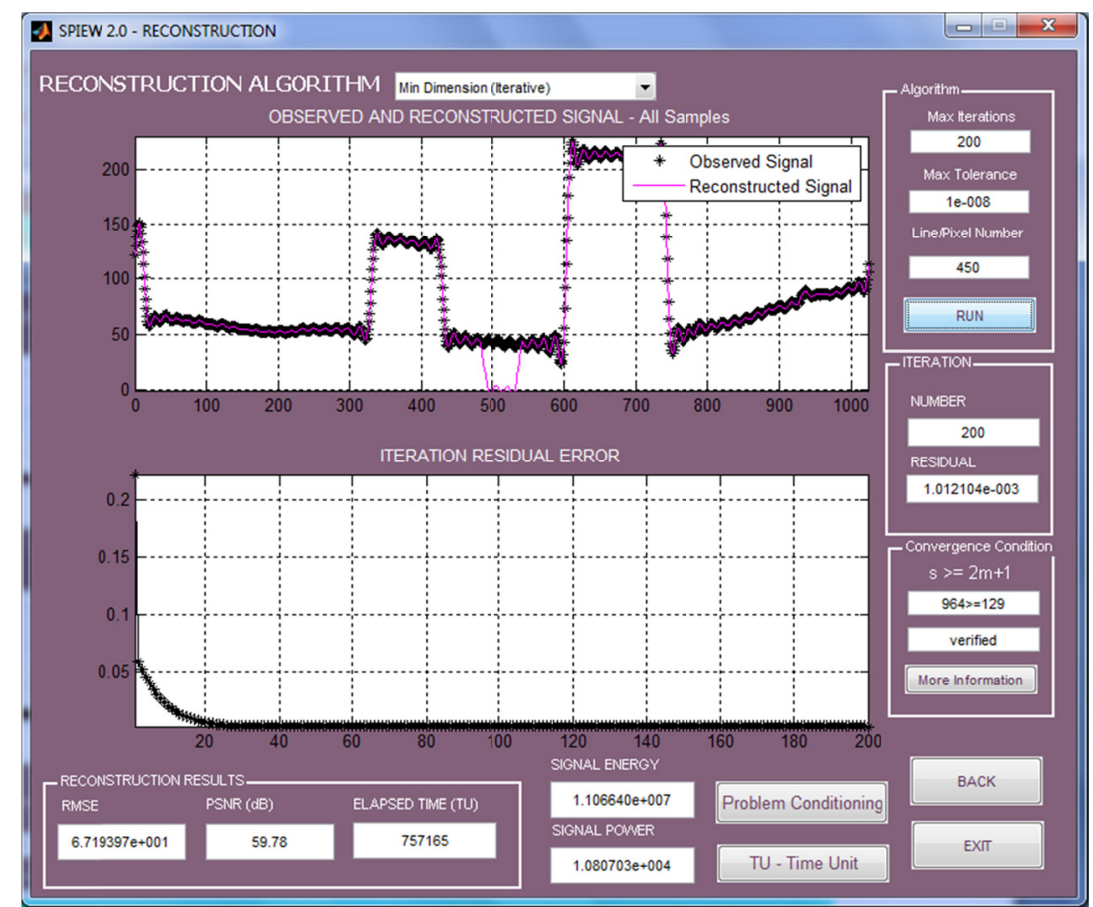

Figure 18a. Reconstruction results using the Minimum Dimension algorithm, Iterative method (Line 450)

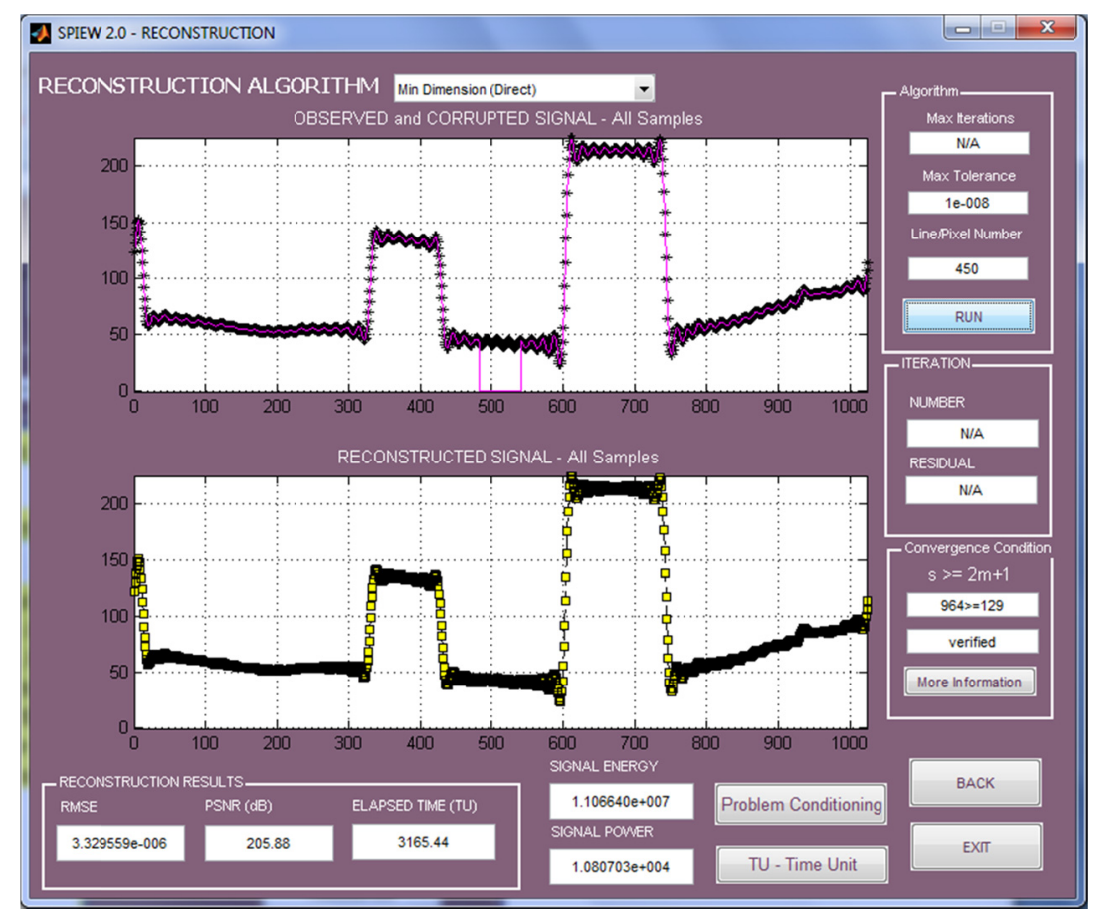

Figure 18b. Reconstruction results using the Minimum Dimension algorithm, Iterative method (Line 450)

In the next case the number of lost samples was increased up to 100. The problem conditioning values are shown in Figure19. As it can be seen in Figure 19, the condition number reached the value of $10^{15}$. Since we used double precision ( 32 bits precision), it is not expected that direct mode can calculate an accurate solution. The computed solution would not be to trust on. Obviously, if we increase the burst size beyond 100, signal remains unrecoverable. Concerning the direct method, as we expected, since we use double precision, the condition number has reached a value of $10^{15}$, and if we increase even more the burst window, the signals cannot be reconstructed and the direct method won't converge to a solution (see Figure 20). 


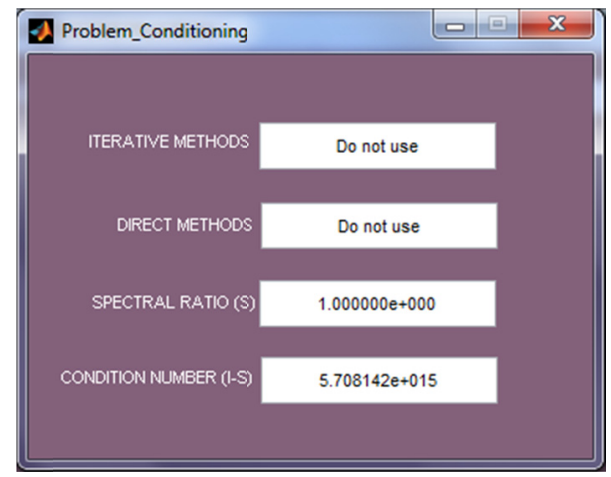

Figure 19. Problem conditioning values for 100 contiguous lost samples

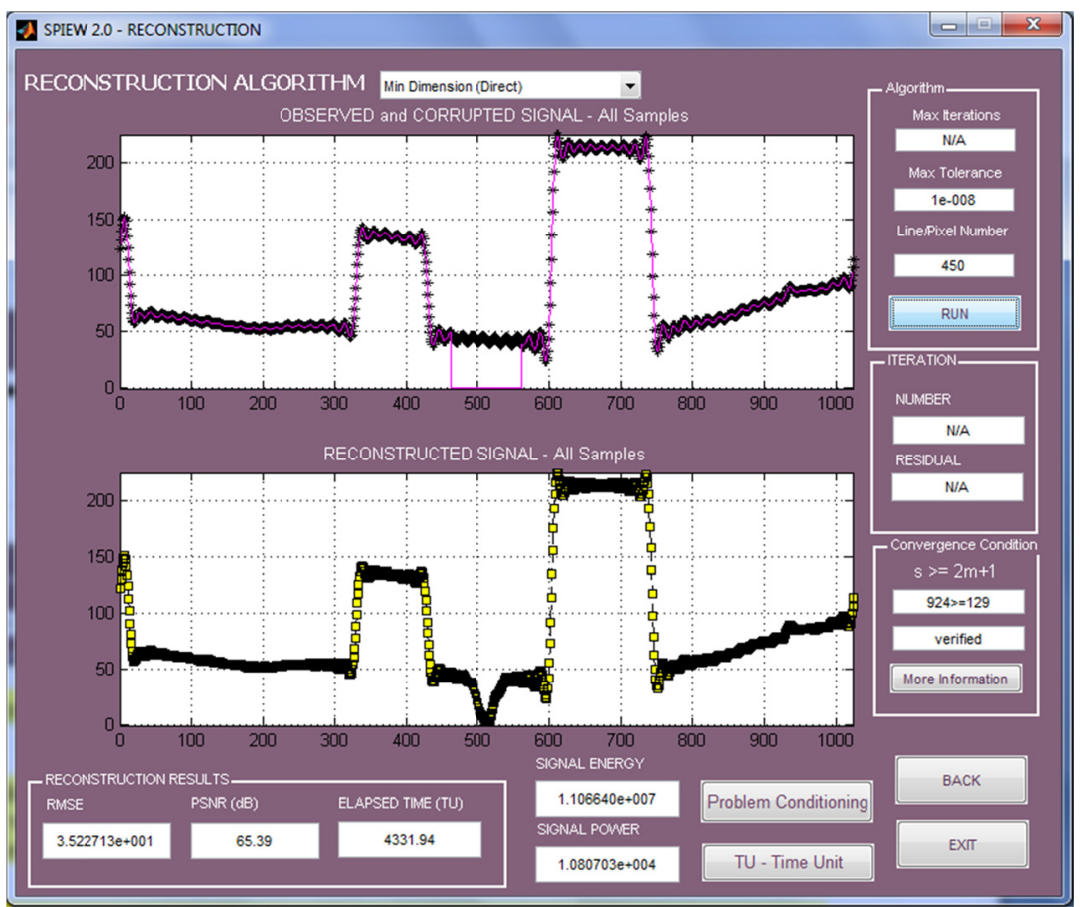

Figure 20. Reconstruction results using the Minimum Dimension Algorithm, Direct method (Line 450)

\section{Survey Results}

After obtaining the limit values to the conditioning of the problem, the tool was presented to a group of test subjects. The tool was properly explained through an oral explanation, and then a written tutorial was given to all of them. Some examples were presented so that they could see the application performance under several different situations.

Thereafter they were asked to explore the tool freely. Afterwards they were given a quiz test to answer. In some issues the test group has shown more difficulty, namely concerning the band limiting operation, as for example its direct relation to the signals redundancy. At the end, trough experimentation, it became clear to them that the decrease of redundancy in the signals clearly limited the number of samples that can be lost.

To validate SPIEW a careful elaboration of the referred quiz test was achieved before it was given to the subjects. In order to be effective, important educational and psychological issues were taken into account on designing it (Minium et al., 1993; Cohen et al., 2000; Caulfield et al., 2011). Seven questions were found as being representatives of the main aspects the tool must provide. Questions are:

(1) "Which oversampling factor would you choose to allow you to lose a larger number of samples in the signals?"

(2) "A high oversampling factor corresponds to a high or low value in the Low Pass Filter Bandwidth?" 
(3) "Which is, in your opinion, the worst error geometry pattern to reconstruct the signals?"

(4) "In a good problem conditioning which reconstruction method would you choose? Why?"

(5) "If the spectral radius of matrix $S$ is smaller than 1, which of the algorithms can you choose to reconstruct the signals?

(6) "If the condition number has a value of $10^{15}$ can you reconstruct the signals using the available methods?"

(7) "Do you consider the tool useful? Did your knowledge in the area improved?"

The results obtained in quiz done to the subjects are showed in Figure 21.

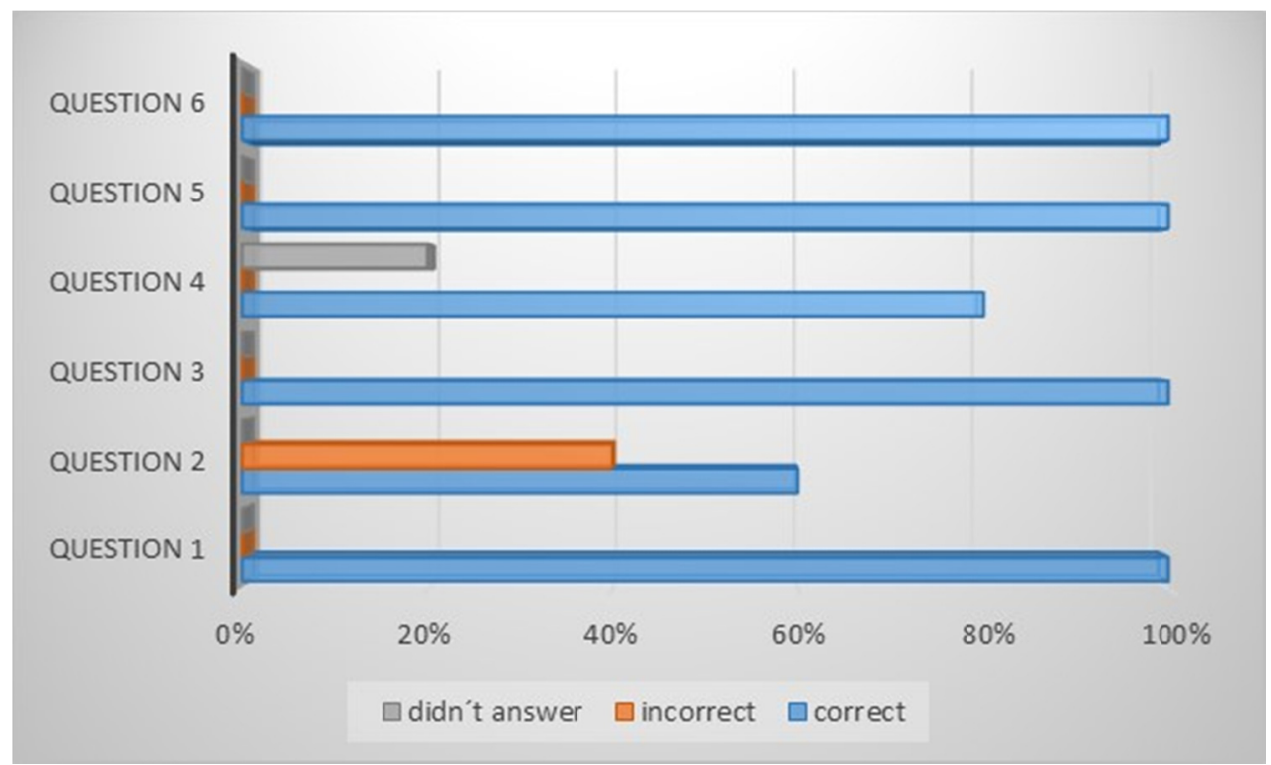

Figure 21. Test results

As it can be seen, $100 \%$ of the subjects answered correctly to questions 1, 3, 5 and $6.20 \%$ didn't answer to question 4 . Concerning question 2, 60\% answered correctly and 40\% incorrectly. Finally, concerning question 7 , they all consider the tool helpful, and say that their knowledge has improved with it.

\section{Conclusions and Future Work}

Similar to its previous version, SPIEW allows indubitably its users to deal with signal reconstruction algorithms in a much friendlier and accessible way. By approaching the problem in a more graphical and interactive manner, the users were able to retain and consolidate concepts such as filtering, band-limiting, problem conditioning, reconstruction and algorithm dimensions. Other concepts like signal redundancy and how it affects the number of samples that can be lost are also addressed in this version of the tool, as well as the three different error geometry patterns available and the identification of the worst case scenario to the reconstruction can also be tested.

Depending on the problem condition analysis the user can know in advance if the signal reconstruction is possible, or which is the best algorithm to reconstruct it and confront its previous hints or results with precise predictions. It permits user to enhance its knowledge and get the feeling about this kind of problems in an interactive way. This analysis is done trough the convergence condition, spectral radius and condition number parameters. This allows the user to alter its problem conditioning to the desired goal before the reconstruction begins.

To compare the results obtained using the three different algorithms available in the tool the RMSE and PSNR of the reconstructed signals are computed. In the previous version of the tool only one reconstruction method was available, the Papoulis-Gerchberg algorithm. The reconstruction time, measured in TU (Time Units) can also be used to compare the performance of the three algorithms. Besides this, in the specific case of the iterative methods, the maximum number of iterations necessary to reconstruct the signal and the graphic evolution of the 
Iteration Residual Error can also act as comparative values between the iterative methods performance. In the direct method, instead of the iteration error evolution graphic, both the reconstruct and corrupted signal versions are shown.

This application is to be used in Digital Signal and Image Processing related subjects. So far, the users' receptivity has been encouraging. Currently we have used in this tool high frame rate sequences and still images obtained with the MVD method (Rusanovskyy et al., 2013), still we believe it can be used in images from different areas, such as medical, e.g.

As future work we aim to add new features to SPIEW (Karthik \& Prabhu, 2010) and conduct more tests with graduated students $(\mathrm{MSc} \& \mathrm{PhD})$. The aim is finding the critical points that establish the appropriate method according to a previous analysis of the problem conditioning.

\section{References}

Caulfield, C., Xia, J., Veal, D., \& Maj, S. P. (2011). A Systematic Survey of Games Used for Software Engineering Education. Modern Applied Science, 5(6), 28-43. http://dx.doi.org/10.5539/mas.v5n6p28

Cohen, L., Manion, L., \& Morrison, K. (2000). Research Methods in Education. Londres: RoutledgeFalmer. http://dx.doi.org/10.4324/9780203224342

Costa, M., Barroso, J., \& Soares, S. (2012). Signal Processing Interpolation Educational Workbench. Computer Applications in Engineering Education, Wiley InterScience, 20(2), 356-363. http://dx.doi.org/10.1002/cae.20402

Ferreira, P. J. S. G. (1994a). Interpolation and the Discrete Papoulis-Gerchberg Algorithm. IEEE Transactions on Signal Processing, 42(10), 2596-2606. http://dx.doi.org/10.1109/78.324726

Ferreira, P. J. S. G. (1994b). Noniterative and fast iterative methods for interpolation and extrapolation. IEEE Transactions on Signal Processing, 42(11), 3278-3282. http://dx.doi.org/10.1109/78.330398

Ferreira, P. J. S. G. (1994c). The Stability of a Procedure for the Recovery of Lost Samples in Band-Limited Signals. IEEE Transactions on Signal Processing, 42(11), 3278-3282. http://dx.doi.org/10.1109/78.330398

Golub, G., \& Van Loan, C. (1996). Matrix computations (3rd ed.). The Johns Hopkins Univ Press.

Jones, M. C. (1986). The Discrete Gerchberg Algorithm. IEEE Transactions on Acoustics Speech and Signal Processing, 3(3), 624-626. http://dx.doi.org/10.1109/TASSP.1986.1164861

Karthik, M., \& Prabhu, K. (2010). On the Eigenvalues of Matrices for the Reconstruction of Missing Uniform Samples. IEEE Transactions on Signal Processing, 58(5), 2896-2900. http://dx.doi.org/10.1109/TSP.2010.2041277

Kincaid, D., \& Cheney, W. (2002). Numerical Analysis: Mathematics of Scientific Computing (3rd ed.). The Brooks/Cole - Thompson Learning.

Matlab. (2012). A product of The MathWorks, Inc. Retrieved from http://www.mathworks.com/

Minium, E. W., King, B. M., \& Bear, G. (1993). Statistical Reasoning in Psychology and Education. New York: John Wiley \& Sons, Inc.

Neves, F., Soares, S., Reis, M. C., Tavares, F., \& Assunção, P. (2008). VoIP reconstruction under a minimum interpolation algorithm. IEEE International Symposium on Consumer Electronics, ISCE 2008. http://dx.doi.org/10.1109/ISCE.2008.4559434

Papoulis, A. (1975). A New Algorithm in Spectral Analysis and Band-Limited Extrapolation. IEEE Transactions on Circuits and Systems, 22(9), 735-742. http://dx.doi.org/10.1109/TCS.1975.1084118

Rusanovskyy, D., Hannuksela, M., \& Su, W. (2013). Depth-based Coding of MVD Data for 3D Video Extension of H.264/AVC, 3D Res. 04. http://dx.doi.org/10.1007/3DRes.02(2013)6

\section{Copyrights}

Copyright for this article is retained by the author(s), with first publication rights granted to the journal.

This is an open-access article distributed under the terms and conditions of the Creative Commons Attribution license (http://creativecommons.org/licenses/by/3.0/). 